\title{
Cardiac Rhythm Vigilance in Ketogenic Diet
}

\author{
Sanjay Kalra, ${ }^{1}$ Kamal Kishor ${ }^{2}$ and Deepak Khandelwal ${ }^{3}$ \\ 1. Department of Endocrinology, Barti Hospital, Karnal, India; 2. Department of Cardiology, Rama Hospital, Karnal, India; 3. Department of \\ Endocrinology, Maharaja Agrasen Hospital, New Delhi, India
}

DOI: https://doi.org/10.17925/EJAE.2018.4.2.51

$\mathrm{K}$ etogenic diet is a popular mode of treatment for weight loss, diabetes and seizures, which must be followed under medical supervision. This brief communication reviews literature related to the pro-arrhythmogenicity of the ketogenic diet. Modern ketogenic diet, followed with attention to electrolyte and mineral balance, is safe, and is not associated with arrhythmias. However, electrocardio vigilance is required while initiating and continuing ketogenic diet.

\section{Keywords}

Arrhythmia, atrial fibrillation, cardiovigilance, diabetes

Disclosure: Sanjay Kalra, Kamal Kishor and Deepak Khandelwal have nothing to declare in relation to this article.

Review Process: Double-blind peer review.

Compliance with Ethics: This study involves a review of the literature and did not involve any studies with human or animal subjects performed by any of the authors.

Authorship: All named authors meet the criteria of the International Committee of Medical Journal Editors for authorship for this manuscript, take responsibility for the integrity of the work as a whole and have given final approval for the version to be published.

open Access Statement: This article is published under the Creative Commons Attribution Noncommercial License, which permits any non-commercial use, distribution, adaptation and reproduction provided the original author(s) and source are given appropriate credit. (c) The Authors 2018

Received: 13 July 2018

Accepted: 31 July 2018

Citation: European Journal of Arrhythmia \&

Electrophysiology, 2018:4(2):51-2

Corresponding Author: Sanjay Kalra, Bharti Hospital, Karnal 132001 India, E: bridekn|@gmail.com

Support: No funding was received in the publication of this article.
The ketogenic diet is experiencing a resurgence in endocrine and metabolic circles, and its use has become popular in recent years. ${ }^{1}$ This encouraging trend stems from two reasons: the ever-increasing burden of the obesity and diabetes pandemics, as well as the lack of results with existing, conventional weight-loss therapy. Both obesity and diabetes are associated with cardiovascular disease. As part of the metabolic syndrome, ${ }^{2}$ these, and other cardiovascular risk factors, need to be handled in a comprehensive manner. This awareness has led to cardiovascular outcome trials being made mandatory for the development of glucose-lowering medication. ${ }^{3}$

\section{Cardiac benefits}

Some concerns have been raised regarding the cardiovascular safety of non-pharmacological weight-lowering interventions such as restricted diets. ${ }^{4}$ The ketogenic diet, too, has been the subject of various safety reviews in the past. 5,6

Ketogenic diet is known to improve cardiovascular risk factors such as weight, glycaemia and lipids. Studies in athletes and in obese persons have shown the maintenance of exercise capacity, after adaptation to a ketogenic source of energy. ${ }^{7.8}$ Apart from the improvement in traditional parameters, a reduction in sympathetic activity and natriuresis have also been reported with ketogenic diet. ${ }^{9}$ These mechanisms, also, may lead to improvement in cardiac parameters.

\section{Cardiac concern}

Paradoxically, however, cardiac complications have been reported in children and adolescents on ketogenic diet. A study of 20 children revealed prolonged QT interval (QTC) and cardiac chamber enlargement in three patients each. A significant correlation between prolonged QTC and both low-serum bicarbonate and high-beta-hydroxybutyrate was noted. One patient with severe dilated cardiomyopathy and prolonged QTC normalised when the diet was discontinued. ${ }^{10}$ Case reports of sudden cardiac death in adolescents have also been published. ${ }^{11,12}$ Yet another case report describes fatality in a 10-year-old child on chronic propofol infusion, who was started on a ketogenic diet. ${ }^{13}$ The propofol infusion syndrome is characterised by metabolic acidosis, lipaemia, rhabdomyolysis and myocardial failure. Propofol interferes with fatty acid oxidation, and may prevent a fat-rich diet from being metabolised. Torsades de pointes has also been reported with ketogenic diet. ${ }^{14}$ These data suggest the need for enhanced cardiovigilance in persons on ketogenic diet.

\section{Rationale}

Various suggestions for the pro-arrhythmogenic effect of ketogenic diet include selenium deficiency, hypokalaemia and alteration in autonomic nervous system activity. It must also be noted that reports of sudden cardiac death were frequent in the past, when liquid protein diets were used. ${ }^{4-6,9,15}$ Modern ketogenic diets, taken under medical supervision, are safe and well tolerated, especially from a cardiac and electrocardiac perspective. However, electrocardiac vigilance must be maintained during such dietary therapy.

\section{Cardiac safety}

Some studies, however, have shown no abnormality in duration of P wave and QT interval in children treated with a ketogenic diet. ${ }^{14,16}$ In a 6-month trial of 25 drug-resistant children with epilepsy treated with a ketogenic diet, electrocardiography was performed at baseline and at 6 months into the ketogenic diet. Heart rate, maximum and minimum P-wave duration, P-wave dispersion, and 
maximum and minimum corrected QT interval and QT dispersion were manually measured from the 12-lead surface electrocardiogram (ECG). Minimum and maximum corrected QT and QT dispersion measurements showed a non-significant increase after 6 months compared with baseline values. No significant changes were observed in other parameters. ${ }^{14}$ In a serial study of 27 children aged 6 months to 5 years with refractory epilepsy, treated with classic ketogenic diet, using a non-fasting gradual initiation protocol, with supplementation with oral calcium and selenium, ECGs were obtained at baseline and after 1, 3, 6 and 12 months. No statistically significant change was noted in the corrected QT interval. No ST segment changes or dysrhythmias were recorded. ${ }^{16}$

While these studies restore confidence in the cardiac safety of ketogenic diet, especially in adults, there is a need for continued vigilance. ${ }^{17}$

\section{Pragmatic vigilance}

The maintenance of optimal fluid electrolyte and mineral status is the key to the prevention of arrhythmias during ketogenic diet. All modern ketogenic diets include adequate hydration and sodium supplementation as a part of their protocol. Micro-nutrient adequacy, especially of selenium, should also be ensured by supplementation. Current screening guidelines call for estimation of baseline and follow-up selenium levels every 3 months during the first year of ketogenic diet, along with recommended daily allowance of selenium supplementation (30 mcg/day). ${ }^{18}$

Since life-threatening arrhythmias may be a reflection of selenium deficiency, we recommend routine screening of ECG prolongation of QT or electric instability at baseline and every 3 months. This frequency is concordant with the guidance related to selenium monitoring. Careful screening and monitoring of symptoms of arrhythmia must be included in the medical supervision of ketogenic diet. ECGs should also be carried out whenever unexplained symptoms occur.

Drugs which may cause QT prolongation or precipitate arrhythmias must be avoided or taken under close supervision. These simple measures should help ensure that the benefits of a ketogenic diet are achieved, while ensuring electrocardiac safety.
1. Gupta L, Khandelwal D, Kalra S, et al. Ketogenic diet in endocrine disorders: current perspectives. J Postgrad Med. 2017;63:242-51

2. Ranasinghe $P$, Mathangasinghe $Y$, Jayawardena $R$, et al. Prevalence and trends of metabolic syndrome among adults in the Asia-Pacific region: a systematic review. BMC Public Health 2017;17:101

3. John M, Gopalakrishnan Unnikrishnan A, Kalra S, Nair T Cardiovascular outcome trials for anti-diabetes medication: a holy grail of drug development? Indian Heart J. 2016;68:564-71.

4. Parikh P, McDaniel MC, Ashen MD, et al. Diets and cardiovascular disease: an evidence-based assessment. J Am Coll Cardiol. 2005;45:1379-87.

5. Bilsborough SA, Crowe TC. Low carbohydrate diets: what are the potential short- and long-term health implications? are the potential short- and long-term
Asia Pac J Clin Nutr. 2003;12:397-404.

6. Crowe TC. Safety of low-carbohydrate diets. Obes Rev. 2005;6:235-45.
7. Phinney SD, Horton ES, Sims EA, et al. Capacity for moderate exercise in obese subjects after adaptation to a hypocaloric, ketogenic diet. J Clin Invest. 1980;66:1152-61.

8. Phinney SD, Bistrian BR, Evans WJ, et al. The human metabolic response to chronic ketosis without caloric restriction: preservation of submaximal exercise capability with reduced carbohydrate oxidation. Metabolism. 1983;32:769-76.

9. DeHaven J, Sherwin R, Hendler R, Felig P. Nitrogen and sodium balance and sympathetic-nervous-system activity in obese subjects treated with a low-calorie protein or mixed diet. N Engl J Med. 1980;302:477-82.

10. Best TH, Franz DN, Gilbert DL, et al. Cardiac complications in pediatric patients on the ketogenic diet. Neurology. 2000:54:2328-30.

11. Bank IM, Shemie SD, Rosenblatt B, et al. Sudden cardiac death in association with the ketogenic diet. Pediatr Neurol. 2008:39:429-31.
12. Stevens A Robinson DP Turpin 1 et al. Sudden cardiac death of an adolescent during dieting South Med I. 2002:95:1047-9.

13. Baumeister FA, Oberhoffer R, Liebhaber GM, et al. Fatal propofol infusion syndrome in association with ketogenic diet. Neuropediatrics. 2004;35:250-2.

14. Doksöz Ö, Güzel O, Yılmaz Ü, et al. Dispersion durations of $\mathrm{P}$-wave and QT interval in children treated with a ketogenic diet. Pediatr Neurol. 2014;50:343-6.

15. Phinney SD, Bistrian BR, Kosinski E, et al. Normal cardiac rhythm during hypocaloric diets of varying carbohydrate content. Arch Intern Med. 1983;143:2258-61.

16. Sharma S, Gulati S. The ketogenic diet and the QT interval. J Clin Neurosci. 2012;19:181-2

17. Wadden TA, Stunkard AJ, Brownell KD. Very low calorie diets: their efficacy, safety, and future. Ann Intern Med. 1983;99:675-84.

18. Sirikonda NS, Patten WD, Phillips JR, Mullett CJ. Ketogenic diet: rapid onset of selenium deficiency-induced cardiac decompensation. Pediatr Cardiol. 2012;33:834-8. 